\title{
Thermal and Rheological Properties of Agarose-Dimethyl Sulfoxide-Water Gels
}

\author{
Mineo WATASE and Katsuyoshi NiSHINARI* \\ Chemical Research Laboratory, Faculty of Liberal Arts, \\ Shizuoka University, Ohya, Shizuoka 422, Japan \\ *Instrumentation Research Laboratory, National Food Research Institute, \\ Ministry of Agriculture, Forestry and Fisheries, Tsukuba 305, Japan
}

(Received June 14, 1988)

\begin{abstract}
DSC and dynamic viscoelastic measurements were carried out for agaroseDMSO-water gels in order to clarify the relation between the structure and gelling properties of agarose. Gelling ability showed a maximum at DMSO concentration of $0.277 \mathrm{~mol}$ fraction (mf). It is suggested that DMSO molecules interact strongly with water molecules and as a result the effective concentration of agarose increases with increasing concentration of DMSO up to $0.277 \mathrm{mf}$. Then, the gelling ability increases with increasing concentration of DMSO. Beyond this concentration of DMSO, the free DMSO, which has high dissolving power, increases, and then the formation of junction zones is inhibited. Thus, the gelling ability decreases with increasing concentration of DMSO. At a concentration of DMSO of $0.277 \mathrm{mf}$, the number and structures of junction zones and the conformation of flexible chain molecules connecting junction zones are optimized to give maxima of the elastic modulus, the melting temperature and endothermic enthalpy of melting. The gel containing $0.277 \mathrm{mf}$ DMSO showed no melting endothermic DSC peak when heated from $-100^{\circ} \mathrm{C}$.
\end{abstract}

KEY WORDS Agarose / Dimethyl Sulfoxide / Differential Scanning Calorimetry / Dynamic Young's Modulus /

Agarose is the main component of agar, and governs the mechanical properties of agar gels. Since agarose is a neutral polysaccharide, the rheological and thermal properties are not affected by cations, contrary to carrageenan. ${ }^{1}$ It was shown that the addition of glycerin or ethylene glycol changes remarkably the thermal and rheological properties of agarose and kappa-carrageenan gels. ${ }^{2}$ Effects of urea, guanidine hydrochloride or formamide on these properties of agarose and kappa-carrageenan gels were also investigated. ${ }^{3}$

Dimethyl sulfoxide (DMSO) is well known as a cryoprotector such as glycerol for cells against freezing damage. ${ }^{4}$ Stress relaxation measurements were carried out on agaroseDMSO-water gels, and the analysis using a 6 element mechanical model consisting of three
Maxwell elements in parallel showed that the elastic modulus of the Maxwell element with the longest relaxation time showed a maximum at a concentration of DMSO mole fraction range from 0.24 to $0.32 .^{5}$ In order to examine the mechanism of this phenomenon, the dynamic mechanical measurements and differential scanning calorimetry of agaroseDMSO-water gels were carried out in the present work.

\section{EXPERIMENTAL}

\section{Materials}

Agarose was extracted from sea weeds, Gelidium amansii produced in Izu (Japan) in 1987. Sea weeds were pretreated with $6 \%$ aqueous solutions of sodium hydroxide at 
about $65^{\circ} \mathrm{C}$. Two fractions of agarose were prepared by extracting at 130 and $132^{\circ} \mathrm{C}$ respectively by the same method described previously. ${ }^{6}$ Characteristics of the two fractions are shown in Table I. The agarose powders were swollen at $40^{\circ} \mathrm{C}$ for $10 \mathrm{~h}$, then preheated at $70^{\circ} \mathrm{C}$ for $30 \mathrm{~min}$, and heated at $100^{\circ} \mathrm{C}$ for $1 \mathrm{~h}$ to attain complete dissolution. The solution was poured into teflon molds and cooled slowly to form gels. They were kept in a refrigerator at $5^{\circ} \mathrm{C}$ for two days, and at the measurement temperature for $1 \mathrm{~h}$ before the measurement was carried out. An extra fine reagent grade of DMSO (Wako Junyaku Kogyo) was used without further purification.

\section{Measurements}

Differential scanning calorimetry (DSC) of gels was carried out on a Sensitive DSC SSC $560 \mathrm{U}$ (Seiko Instruments \& Electronics, Ltd.). Forty five mg each of the gels were sealed in a silver pan of $70 \mu \mathrm{l}$. Distilled water was used as the reference, and the weight was made equal within $\pm 0.1 \mathrm{mg}$ to that of the sample gel in order to obtain a flat base line. The temperature was raised from $2^{\circ} \mathrm{C}$ at the heating rate of $2^{\circ} \mathrm{C} \mathrm{min}{ }^{-1}$ to observe an endothermic peak accompanying the transition from gel to sol. In order to examine this transition, the temperature was kept constant for 10 min after melting, and then lowered at the cooling rate of $2^{\circ} \mathrm{C} \min ^{-1}$.

Low temperature DSC was carried out in order to examine the state of water in agarose gels. $15 \pm 0.1 \mathrm{mg}$ of gel samples were sealed in aluminium pans of $15 \mu \mathrm{l}$. Alpha alumina was used as the reference. The temperature was lowered rapidly by liquid nitrogen from the ambient one to $-100^{\circ} \mathrm{C}$, kept at that temperature for $10 \mathrm{~min}$, and then raised at the rate of $4^{\circ} \mathrm{C} \min ^{-1}$.

The dynamic Young's modulus $E^{\prime}$ and mechanical loss tangent $\tan \delta$ were measured under longitudinal vibration of a cylindrically molded gel $(20 \mathrm{~mm}$ diameter and $30 \mathrm{~mm}$ length) at $2.5 \mathrm{~Hz}$. The temperature of the gel
Table I. Characterization of agarose samples

\begin{tabular}{cccc}
\hline Agarose & $\begin{array}{c}\text { Intrinsic } \\
\text { viscosity }\end{array}$ & $\begin{array}{c}\text { 3,6-Anhydro- } \\
\text { L-galactose } \\
\text { content }^{\mathrm{b}}\end{array}$ & $\begin{array}{c}\text { Sulfate } \\
\text { content }^{\mathrm{c}} \\
(\mathrm{mol} /\end{array}$ \\
$\mathrm{nl} \mathrm{g}{ }^{-1}$ & $-\frac{\left.\mathrm{C}_{6} \mathrm{H}_{10} \mathrm{O}_{5}\right)}{\%}$ & 0.002 \\
$\mathrm{~F} 1$ & 1.7 & 42.2 & 0.003 \\
\hline
\end{tabular}

a The intrinsic viscosity was determined in $0.1 N \mathrm{KSCN}$ solution at $35^{\circ} \mathrm{C}$.

b 3,6-Anhydro-L-galactose content was determined by the quantitative colorimetric method using fructose as the standard.

c Sulfate content was determined by elemental analysis of a powdered specimen.

was controlled by a silicone oil bath. The apparatus used was a Rheolograph Gel from Toyo Seiki Seisakusho Ltd.

\section{RESULTS}

Heating (a, b, c, d, e) and cooling $\left(\mathrm{a}^{\prime}, \mathrm{b}^{\prime}, \mathrm{c}^{\prime}\right.$, $\left.d^{\prime}, e^{\prime}\right)$ DSC curves are shown for $F 1$ agarose gels of various concentrations (Figure 1(A)) and those containing $0.04 \mathrm{~mol}$ fraction (mf) DMSO (Figure 1(B)). Endothermic peaks in the heating curves are attributed to the transition from gel to sol or the melting of gels, and the peak temperature will be called the melting temperature $T_{\mathrm{m}}$ hereafter. Exothermic peaks in the cooling curves are attributed to the transition from sol to gel, and the peak temperature will be called the setting temperature $T_{\mathrm{s}}$ hereafter. $T_{\mathrm{m}}$ and $T_{\mathrm{s}}$ of agarose gels without DMSO shifted to higher temperatures with increasing concentration of agarose (Figure 1(A)). While $T_{\mathrm{m}}$ of agarose gels containing $0.04 \mathrm{mf}$ DMSO shifted to higher temperatures with increasing concentration of agarose up to $10 \%(\mathrm{w} / \mathrm{w})$, it shifted to a lower temperature for the $15 \%(\mathrm{w} / \mathrm{w})$ agarose gel (Figure 1(B)). Figures 2 and 3 show heating and cooling DSC curves for F1 agarose gels whose concentrations are the same as in Figure 1, but with higher $\mathrm{mf}$ of DMSO. In the agarose-DMSO- 


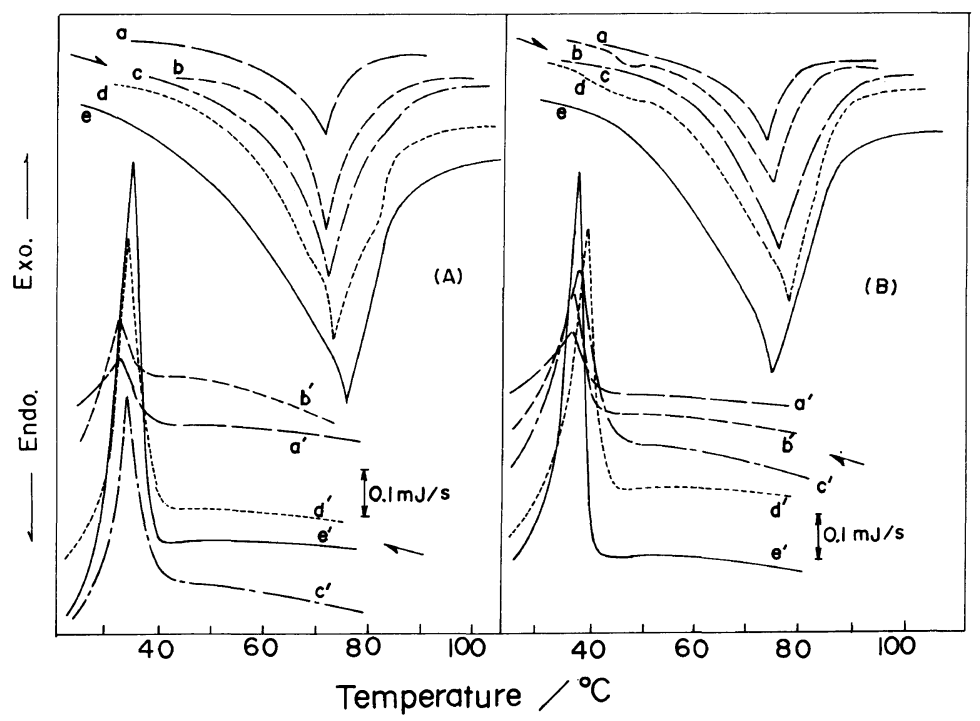

Figure 1. Heating (a, b, c, d, e) and cooling (a', b', $\left.c^{\prime}, d^{\prime}, e^{\prime}\right)$ DSC curves for F1 agarose-DMSO-water gels of various concentrations. (A), without DMSO; (B), $0.04 \mathrm{mf}$ DMSO. Concentration of agarose: $\mathrm{a}, \mathrm{a}^{\prime}$, $2 \%(w / w) ; b, b^{\prime}, 4 \%$ (w/w); c, c $c^{\prime}, 7 \%(w / w) ; d, d^{\prime}, 10 \%(w / w) ; e, e^{\prime}, 15 \%(w / w)$.

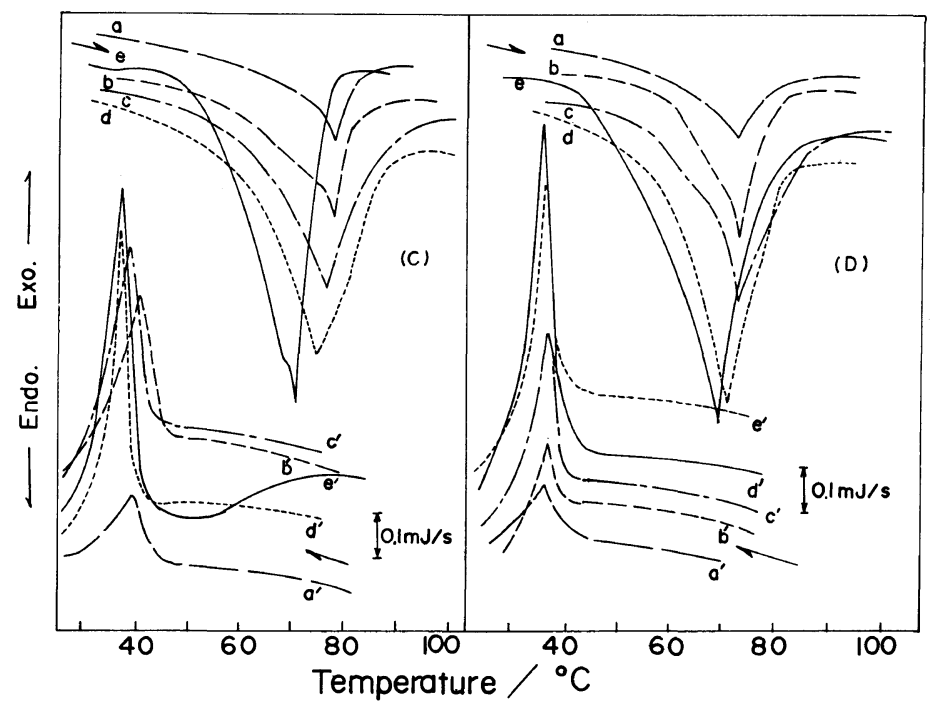

Figure 2. Heating (a, b, c, d, e) and cooling ( $\left.a^{\prime}, b^{\prime}, c^{\prime}, d^{\prime}, e^{\prime}\right)$ DSC curves for F1 agarose-DMSO-water gels of various concentrations. (C), $0.104 \mathrm{mf}$ DMSO; (D), $0.188 \mathrm{mf}$ DMSO. Concentration of agarose is represented in the same way as in Figure 1.

water gels shown in Figure 1(B), Figure 2, and Figure $3, T_{\mathrm{m}}$ and $T_{\mathrm{s}}$ are not monotonously increasing functions of the agarose concentration as in agarose-water gels shown in Figure $1(\mathrm{~A})$. The difference $\Delta T=T_{\mathrm{m}}-T_{\mathrm{s}}$ for agarose gels decreased remarkably with increasing DMSO concentration up to $0.31 \mathrm{mf}$, and beyond that concentrations, DMSO $0.61 \mathrm{mf}$ it showed again a large value. It must be noted that the thermal histories are quite 


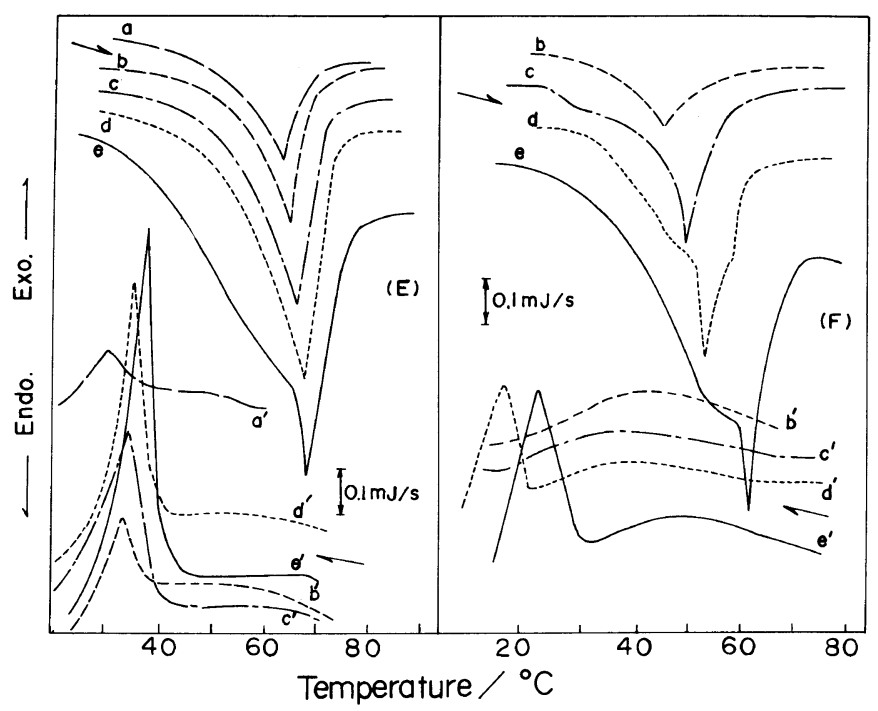

Figure 3. Heating (a, b, c, d, e) and cooling ( $\left.a^{\prime}, b^{\prime}, c^{\prime}, d^{\prime}, e^{\prime}\right)$ DSC curves for F1 agarose-DMSO-water gels of various concentrations. (E), $0.31 \mathrm{mf} \mathrm{DMSO;} \mathrm{(F),} 0.61 \mathrm{mf}$ DMSO. Concentration of agarose is represented in the same way as in Figure 1.

different for heating DSC curves and for cooling DSC curves: gels are kept at $5^{\circ} \mathrm{C}$ for a long time, while they were maintained only for $10 \mathrm{~min}$ after melting for the measurement of cooling DSC curves. Reheating DSC curves did not show sharp endothermic peaks as shown in Figures $1-3$ but only broad endotherms when the scan was made after the temperature was kept constant only for a few hours (results not shown).

Figure 4 shows heating and cooling DSC curves of $10 \%$ (w/w) F2 agarose gels containing various $\mathrm{mfs}$ of DMSO.

Figure 5 shows the dependence of $T_{\mathrm{m}}$ on the concentration of DMSO for F1 agarose gels of various concentrations.

Figure 6 shows the dependence of $T_{\mathrm{m}}$ on the concentration of DMSO for $10 \% \mathrm{w} / \mathrm{w} \mathrm{F1}$ (open circles) and F2 (closed circles) agarose gels.

Figure 7 shows the endothermic enthalpy $\Delta H$ determined from the area surrounded by an endothermic curve and the baselines shown in Figures 1, 2, and 3, as a function of DMSO concentration in mole fraction $(\mathrm{mf})$ for various concentrations of agarose gels ranging from $2 \%(\mathrm{w} / \mathrm{w})$ to $15 \%(\mathrm{w} / \mathrm{w})$.

Figure 8 shows the endothermic enthalpy $\Delta H$ for $10 \%(\mathrm{w} / \mathrm{w})$ agarose gels containing DMSO as a function of DMSO concentration in $\mathrm{mf}$ for $\mathrm{F} 1$ and $\mathrm{F} 2$ fractions.

Dynamic Young's modulus $E^{\prime}$ and mechanical loss tangent $\tan \delta$ of $4 \%$ (w/w) F1 agarose gels containing DMSO are shown as functions of temperature for various concentrations of DMSO in Figure 9. $E^{\prime}$ increased up to about $25^{\circ} \mathrm{C}$ and then decreased with increasing temperature except for the gel with the highest content of DMSO (0.48 mf). Dynamic Young's modulus $E^{\prime}$ of the agarose gels showed a maximum at a DMSO concentration between $0.188 \mathrm{mf}$ and $0.31 \mathrm{mf}$.

In order to examine the influence of the interactions of water with DMSO on gel properties, differential scanning calorimetry was carried out at lower temperatures for $15 \%$ (w/w) F1 agarose gels with various DMSO concentrations. Heating DSC curves showed endothermic peaks accompanying the melting of frozen DMSO-water mixture (Figure 10). 


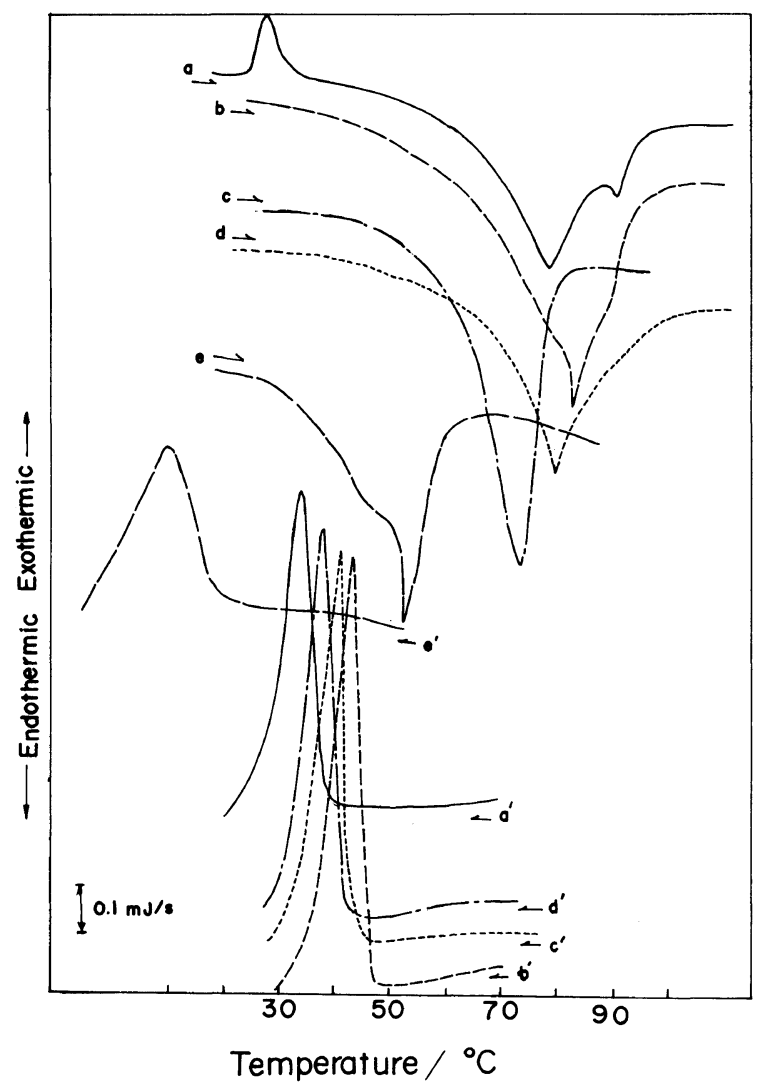

Figure 4. Heating (a, b, c, d, e) and cooling $\left(a^{\prime}, b^{\prime}, c^{\prime}, d^{\prime}, e^{\prime}\right)$ DSC curves of $10 \%(w / w)$ F2 agarose gels containing DMSO. DMSO concentration: a, a', $0 \mathrm{mf}$ DMSO; b, b', $0.095 \mathrm{mf}$ DMSO; c, c', $0.188 \mathrm{mf}$ DMSO; d, d', $0.277 \mathrm{mf}$ DMSO; e, e', $0.450 \mathrm{mf}$ DMSO.

The peak temperature $T_{\text {m.f. }}$ shifted to lower temperatures and the endothermic enthalpy $\Delta H_{\text {m.f. }}$ decreased with increasing DMSO concentrations up to $\mathrm{DMSO}=0.277 \mathrm{mf}$. Above the DMSO concentration $0.277 \mathrm{mf}$, the peak temperature shifted to higher temperatures and the endothermic enthalpy increased with increasing DMSO concentration. The agaroseDMSO-water system with $\mathrm{DMSO}=0.277 \mathrm{mf}$ did not show any endothermic peak. It is worth mentioning that no endothermic peak was observed; i.e., DSC curves were almost flat, for agarose-DMSO-water systems at the neighborhood of DMSO concentration 0.277 $\mathrm{mf}$ although not all these DSC curves are shown here.

\section{DISCUSSION}

It is generally accepted that gelation of agarose occurs in two steps: (i) Lowering the temperature of agarose solution, double stranded helices are formed, and (ii) further cooling produces the aggregation of these helices to form junction zones. ${ }^{7)}$ Therefore, this gelation process corresponds to the cooling DSC curves for the formation of double helices and their aggregation. On the other hand, heating DSC curves correspond to the decomposition of junction zones and the transition from helix to coil.

Agarose is easily dissolved in both water and DMSO. An aqueous solution of agarose form 


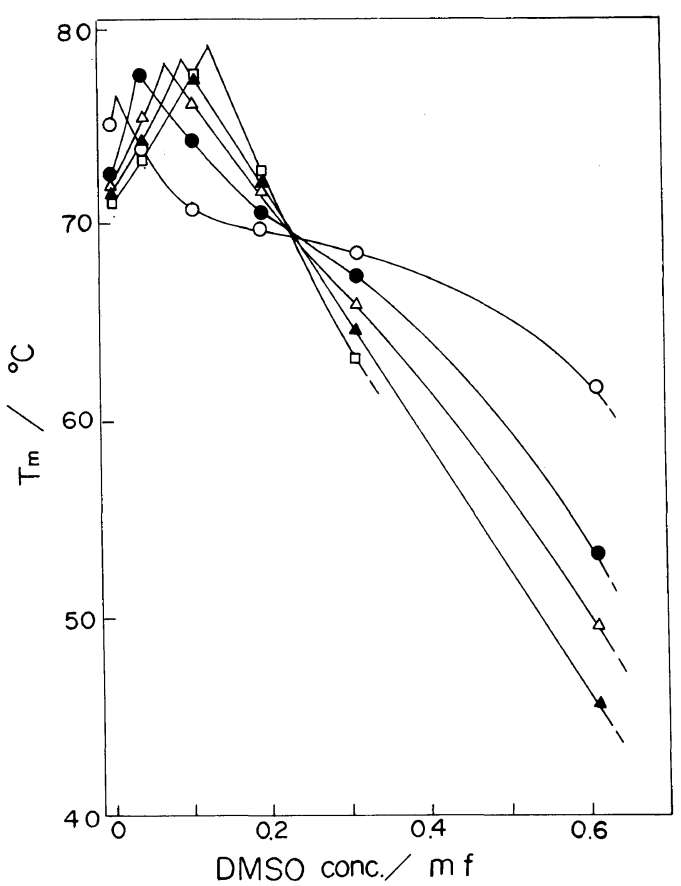

Figure 5. Melting points $T_{\mathrm{m}}$ of $\mathrm{Fl}$ agarose-DMSOwater gels of various concentrations as a function of DMSO concentration. Concentrations of agarose: $\square$, $2 \%(\mathrm{w} / \mathrm{w}) ; \Delta, 4 \%(\mathrm{w} / \mathrm{w}) ; \triangle, 7 \%(\mathrm{w} / \mathrm{w}) ; \bigcirc, 10 \%(\mathrm{w} / \mathrm{w}) ;$ $\bigcirc, 15 \%(w / w)$.

a gel, but a DMSO solution of agarose does not form a gel at room temperatrue. Since the interaction between water and DMSO is very strong, the mixture of water and DMSO shows peculiar characteristics. The structure of a hydrated compound of DMSO has been shown to be $\left(\mathrm{CH}_{3}\right)_{2} \mathrm{SO} \cdot 2 \mathrm{H}_{2} \mathrm{O}$ by thermodynamic and NMR investigations. ${ }^{8}$ However, other research has shown it to be $\left(\mathrm{CH}_{3}\right)_{2} \mathrm{SO} \cdot 3 \mathrm{H}_{2} \mathrm{O}^{9} \mathrm{On}$ adding water to an agarose-DMSO system, which does not form a gel, gelation occurs when the water content exceeds a certain value.

The maxima of the melting temperatures $T_{\mathrm{m}}$ and of the endothermic enthalpies $\Delta H$ for agarose-water-DMSO gel as a function of DMSO concentration may be interpreted as follows. The content of free water which does not interact so strongly with DMSO decreased with increasing DMSO concentration. Therefore, DMSO plays the part of a thickener

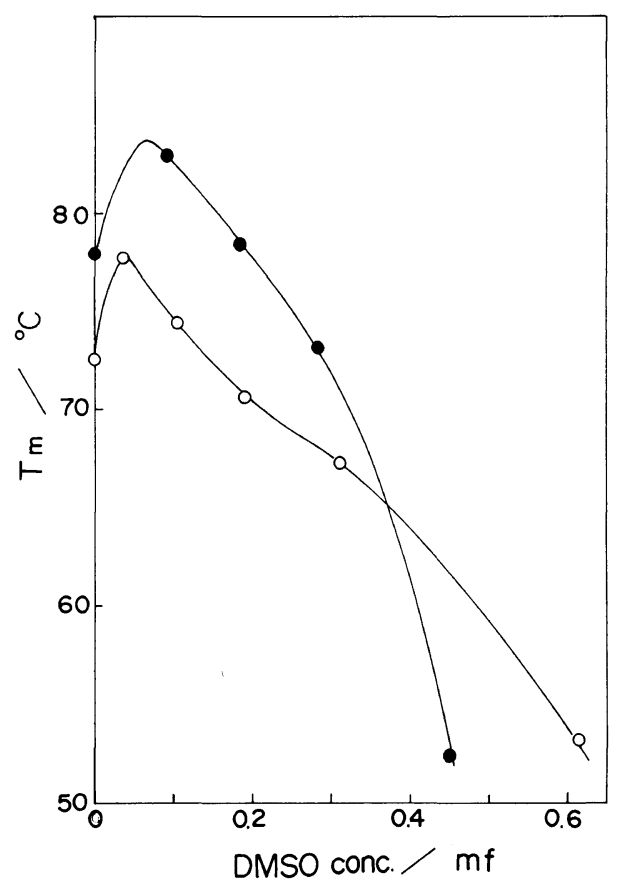

Figure 6. Melting points $T_{\mathrm{m}}$ of $10 \%(\mathrm{w} / \mathrm{w})$ agaroseDMSO-water gels as a function of DMSO concentration. $\mathrm{O}, \mathrm{F} 1 \mathrm{gel} ; \mathrm{O}, \mathrm{F} 2$ gel.

so that the concentration of agarose may increase and as a result, $T_{\mathrm{m}}$ and $\Delta H$ increase with increasing concentration of DMSO. It is well known that many polysaccharide gels consist of junction zones which are somewhat crystalline and of long flexible chains surrounded by a solvent which is amorphous. Thus, the gel is formed by a balance between crystallinity and solubility. This balance shifts to the solubility side at higher concentrations of DMSO than certain values from $0.01 \mathrm{mf}$ to $0.15 \mathrm{mf}$ because of the high dissolving power of DMSO. It is considered that agarose molecules cannot form junction zones in DMSO, while they form junction zones in water. For higher concentrations of agarose, water molecules which are necessary for agarose molecules to form junction zones become insufficient. Therefore, the DMSO concentration at which $T_{\mathrm{m}}$ becomes maximum shifts to lower DMSO concentrations with increasing concentration of agarose as shown in Figure 5. 


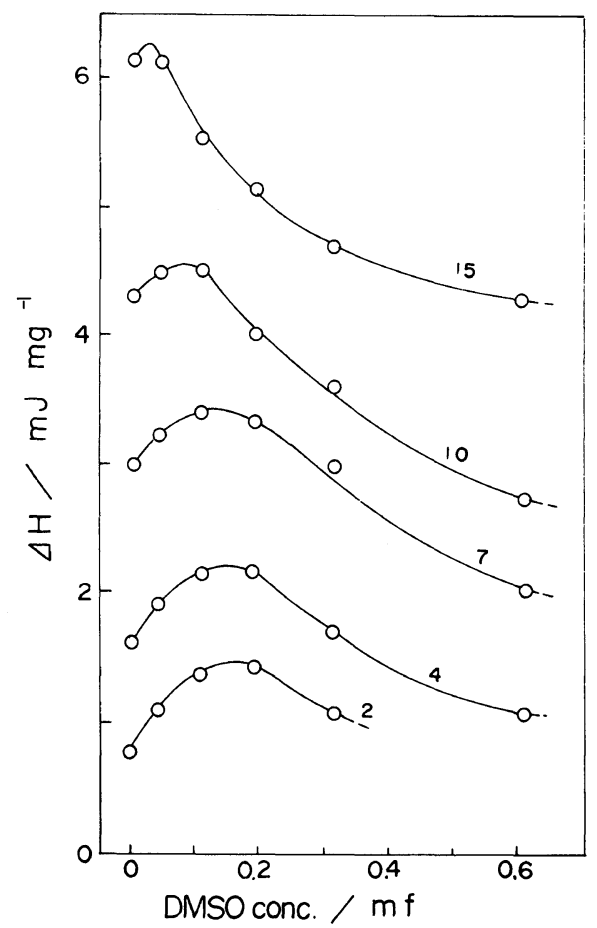

Figure 7. Endothermic enthalpy $\Delta H$ of $\mathrm{F} 1$ agaroseDMSO-water gels as a function of DMSO concentration. Numbers beside each curve denote the concentrations of agarose in $\%(w / w)$.



Figure 8. Endothermic enthalpy $\Delta H$ of $10 \%(\mathrm{w} / \mathrm{w})$ agarose-DMSO-water gels as a function of DMSO concentration. $\mathrm{O}, \mathrm{F} 1 \mathrm{gel} ; \mathbf{O}, \mathrm{F} 2$ gel.

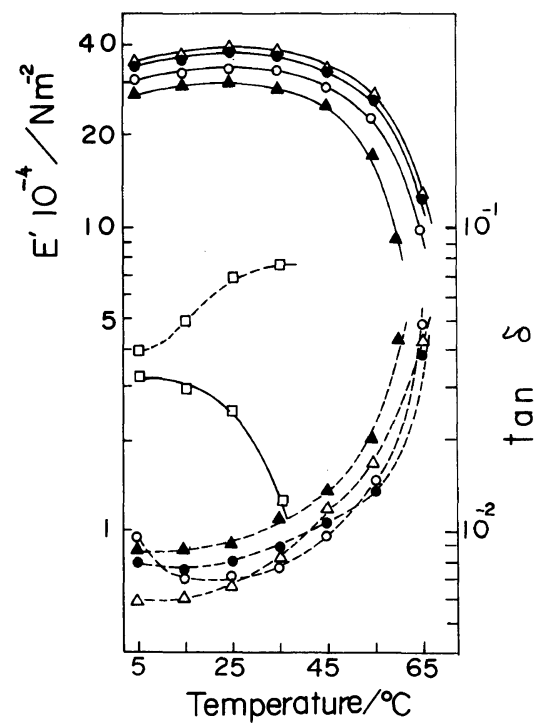

Figure 9. Tepperature dependence of dynamic Young's modulus $E^{\prime}$ (solid line) and $\tan \delta$ (broken line) for $4 \%$ (w/w) F1 agarose-DMSO-water gels. $\bigcirc$, without DMSO; $0.104 \mathrm{mf}$ DMSO; $\triangle, 0.188 \mathrm{mf}$ DMSO; $0.31 \mathrm{mf}$ DMSO; $\square, 0.480 \mathrm{mf}$ DMSO.

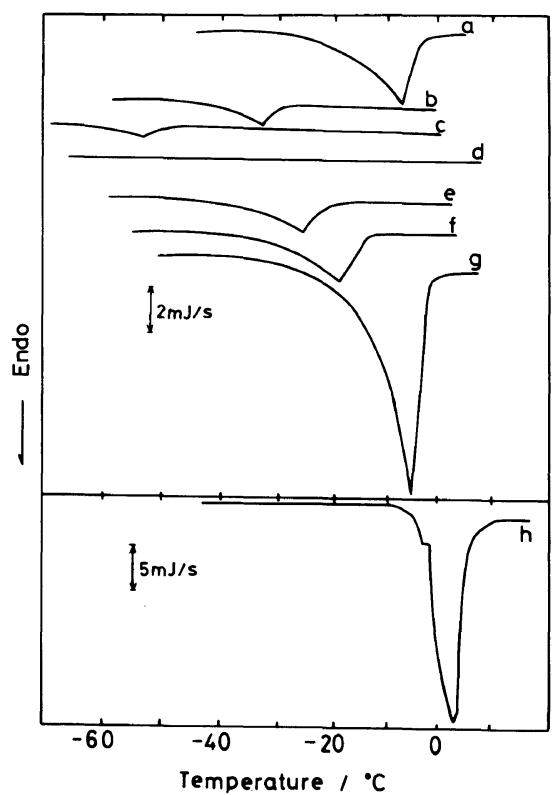

Figure 10. Heating DSC curves of $15 \%(w / w)$ frozen agarose-DMSO-water gels. Heated from $-100^{\circ} \mathrm{C}$ at $4^{\circ} \mathrm{C} \min ^{-1}$. a, $1 \mathrm{mf}$ DMSO; b, 0.61 mf DMSO; c, $0.48 \mathrm{mf}$ DMSO; d, $0.277 \mathrm{mf}$ DMSO; e, $0.188 \mathrm{mf}$ DMSO; f, $0.104 \mathrm{mf}$ DMSO; g, $0.054 \mathrm{mf}$ DMSO; h, without DMSO. 
The ratio of water to DMSO affects strongly the gelling ability of agarose.

The experimental fact that DMSO concentration at which $\Delta H$ becomes maximum shifted to lower DMSO concentrations with increasing concentration of agarose as shown in Figure 7 can be understood by the same way for the case of $T_{\mathrm{m}}$ in Figure 5. $T_{\mathrm{m}}$ shifted to higher temperatures slightly with increasing heating rate, while $T_{\mathrm{s}}$ shifted to lower temperatures with increasing cooling rate in agarosewater system without DMSO. These observations are consistent with general knowledge of thermal analysis. Therefore, $\Delta T=T_{\mathrm{m}}-T_{\mathrm{s}}$ became larger with increasing scanning rate. However, the results shown here were obtained at a scanning rate of only $2^{\circ} \mathrm{C} \mathrm{min}-1$ because it is the most appropriate for experiments. When the scanning rate was much slower than this, it became difficult to obtain clear peaks. In the presence of DMSO 0.61 $\mathrm{mf}$, the gelation was inhibited by free DMSO and then $T_{\mathrm{s}}$ shifted to much lower temperatures than in the case of agarose-water system without DMSO, i.e., about $20^{\circ} \mathrm{C}$ in case of 10 and $15 \%$ agarose gels. This means that gelation takes longer and occurs only at quite low temperatures in the presence of free DMSO than in the case of agarose-water system without DMSO.

The fact that $E^{\prime}$ of agarose gels containing DMSO showed a maximum at the DMSO concentration between $0.188 \mathrm{mf}$ and $0.31 \mathrm{mf}$ (Figure 9) is understood by the same reason. The elastic modulus of thermo-reversible gels depends on the number and structures of cross-linking junction zones, and on the conformation of flexible chains connecting these zones. A simple gel model, consisting of Langevin chains whose both ends are loosely fixed in crystalline junction regions in such a way that the segments near each end are bound densely in one of the junction region, has been proposed in order to explain the temperature dependence of elasticity of thermo-reversible gels. ${ }^{10}$ According to this model, the segments are assumed to be released from the junction zone with increasing temperature just as thread is released from a reel. When all the segments in one of both ends are liberated from the junction zone, the chain is called a free chain in the theory of rubber elasticity, ${ }^{11}$ and ceases to contribute to the elasticity. Using this model, the elastic modulus of thermo-reversible gels can be calculated numerically by assuming the number $\mathscr{N}$ of molecular chains in a unit volume, the values of bonding energy $\varepsilon$, average end-to-end distance of Langevin chains $r_{\mathrm{m}}$, an upper limit $v$ of the number of segments released from the junction zone before the chain ceases to contribute to the elasticity. The number of cross-linking junction zones will decrease with increasing DMSO content. The elastic modulus of thermo-reversible gels is proportional to the number of cross-linking junction zones if the conformation of flexible chains is the same. The presence of DMSO changes the ratio of entropic and energetic contributions to the elastic modulus. The entropic term increases with increasing length of the flexible chains. ${ }^{12}$ At a certain DMSO concentration between 0.188 and $0.31 \mathrm{mf}$, three main factors, the number and structures of cross-linking junction zones, the conformation of flexible chains connecting junction zones, or $\varepsilon, r_{\mathrm{m}}, v$, $\mathscr{N}$ in the above-mentioned model which determine the elastic modulus become optimum to realize the maximum of elastic modulus. The effects of DMSO on the conformation of agarose molecules in water, and on the structures of junction zones should be determined so that we may be able to understand further details in the relation between structure and gelling properties.

Heat of fusion $\Delta H_{\text {m.f. }}$ of $15 \%$ frozen agarose gels together with the endothermic peak temperature $T_{\text {m.f. }}$ of $15 \%$ frozen agarose gels are shown in Figure 11. The endothermic peak area is proportional to the enthalpy related to the melting of frozen solvent. The heat of fusion $\Delta H_{\text {m.f. }}$ of agarose gels was calculated 


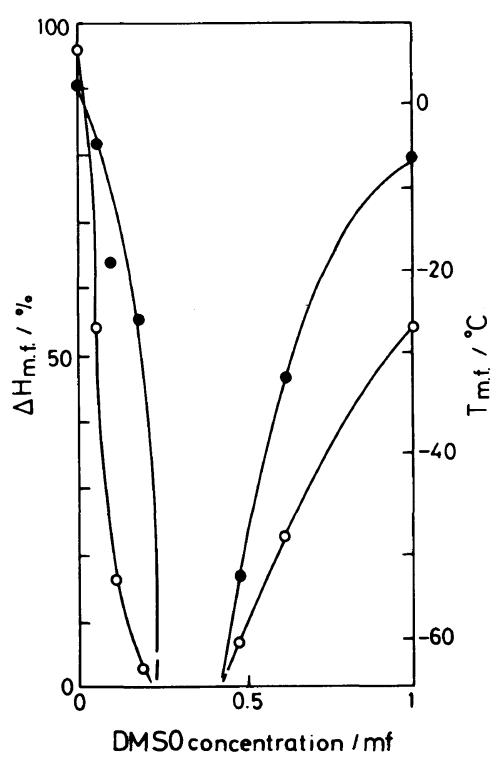

Figure 11. Heat of fusion $\Delta H_{\text {m.f. }}(\bigcirc)$ and $T_{\text {m.f. }}(\bigcirc)$ of $15 \%(w / w)$ frozen agarose-DMSO-water gels as a function of DMSO concentration.

from the area surrounded by the endothermic peak and the base line shown in Figure 10, assuming the heat of fusion for water as 6.008 $\mathrm{kJ} \mathrm{mol}^{-1}$ and that for DMSO as $13.94 \mathrm{~kJ}$ $\mathrm{mol}^{-1}$. Here, it is assumed that below 0.277 mf DMSO, only free water freezes, and that above $0.277 \mathrm{mf}$ DMSO, only free DMSO freezes. The estimated contents of freezing solvent and of non-freezing solvent in $15 \%$ F1 agarose gels are shown in Table II with the melting points for various DMSO concentrations. The content of non-freezing solvent is maximum at a concentration range near $0.277 \mathrm{mf}$. This is due to the strong interaction between water and DMSO, and is consistent with other researches on the hydration of DMSO. ${ }^{8,9}$ The DMSO concentration $0.277 \mathrm{mf}$ is intermediate between the hydrated compounds mentioned above; $\left(\mathrm{CH}_{3}\right)_{2} \mathrm{SO} \cdot 2 \mathrm{H}_{2} \mathrm{O}$ or $\left(\mathrm{CH}_{3}\right)_{2} \mathrm{SO} \cdot 3 \mathrm{H}_{2} \mathrm{O}$. The interaction of water with DMSO is strongest at a DMSO concentration of about $0.277 \mathrm{mf}$. The fact that there appeared no endothermic peak for the system with $0.277 \mathrm{mf}$ DMSO is attributed to the strong interaction of water with
Table II. Contents of freezing solvent and nonfreezing solvent in $15 \%(\mathrm{w} / \mathrm{w}) \mathrm{F} 1$ agaroseDMSO-water gels for various DMSO concentrations ${ }^{\mathrm{a}}$

\begin{tabular}{|c|c|c|c|}
\hline $\begin{array}{l}\text { DMSO } \\
\text { conc }\end{array}$ & $\begin{array}{c}\text { Freezing } \\
\text { solvent }\end{array}$ & $\begin{array}{c}\text { Non-freezing } \\
\text { solvent }\end{array}$ & $T_{\mathrm{m}}$ \\
\hline $\mathrm{mf}$ & $\%$ & $\%$ & ${ }^{\circ} \mathrm{C}$ \\
\hline 0 & 96.0 & 4 & -3.0 \\
\hline 0.054 & 54.1 & 45.9 & -4.7 \\
\hline 0.104 & 16.4 & 83.6 & -18.9 \\
\hline 0.188 & 2.5 & 97.5 & -25.5 \\
\hline 0.277 & 0 & 100.0 & - \\
\hline 0.485 & 6.7 & 93.3 & -52.6 \\
\hline 0.610 & 21.5 & 78.5 & -32.7 \\
\hline 1 & 55.5 & 44.5 & -6.3 \\
\hline
\end{tabular}

a It is assumed that only water freezes below the DMSO concentration $0.277 \mathrm{mf}$, and that only DMSO freezes above the DMSO concentration $0.277 \mathrm{mf}$.

DMSO. This means that there is no freezable water or DMSO observed in the present experimental condition even at $-100^{\circ} \mathrm{C}$ for this system with $0.277 \mathrm{mf}$ DMSO. This is peculiar to DMSO and is not found for other organic solvents as far as the authors know.

\section{REFERENCES}

1. M. Watase and K. Nishinari, Polym. J., 18, 1017 (1986).

2. K. Nishinari and M. Watase, Agric. Biol. Chem., 52, 3231 (1987).

3. M. Watase and K. Nishinari, Food Hydrocolloids, 1, 25 (1986).

4. P. Boutron and A. Kaufmann, Cryobiology, 15, 93 (1978).

5. M. Watase and K. Arakawa, Nippon Kagaku Kaishi, 1353 (1973).

6. M. Watase and K. Nishinari, Rheol. Acta, 22, 580 (1983).

7. D. A. Rees, Pure Appl. Chem., 53, 1 (1981).

8. J. M. G. Cowie and P. M. Toporowske, Can. J. Chem., 39, 2240 (1961).

9. D. H. Rasmussen and A. P. MacKenzie, Nature, 220, 1315 (1968).

10. K. Nishinari, S. Koide; and K. Ogino, J. Phys. (Paris), 46, 793 (1985).

11. L. R. G. Treloar, "The Physics of Rubber Elasticity," Clarendon Press, Oxford, 1975.

12. K. Nishinari, M. Watase, and K. Ogino, Makromol. Chem., 185, 2663 (1984). 